\title{
Area calculation of the level region which is enclosed by the graphical representation of three continuous functions.
}

\author{
George Stathopoulos \\ (Professor of mathematics, Athens, Greece,)
}

Abstract: This proof was conceived in order to introduce a different way of calculating a level region. By the end of the paper I will calculate the area of a level region using continuous functions.

Keywords: calculate, area, enclosed, level, region

\section{Introduction}

As known the area for the level region $(\Omega)$ that we examine is given by a formula, thus a figure is required "necessarily". Enough of my students have asked me if there was a way to avoid signing the functions and drawing a figure to calculate the area. For that reason, I thought of the work that follows, which calculates the area for the region in question without determining the sings of the functions or drawing a figure.

\section{Area calculation of the level region $(\Omega)$}

In the international literature, to my knowledge, the previous area is given by the formula:

$$
E(\Omega)=\int_{\rho_{1}}^{\rho_{2}}\left|f_{3}(x)-f_{1}(x)\right| d x+\int_{\rho_{2}}^{\rho_{3}}\left|f_{3}(x)-f_{2}(x)\right| d x
$$

where $\mathrm{p}_{1}, \mathrm{p}_{2}, \mathrm{p}_{3}$ are the roots of the equations: $f_{1}(x)=f_{3}(x), f_{1}(x)=f_{2}(x), f_{2}(x)=f_{3}(x)$ respectively and for the calculation of $\mathrm{E}(\Omega)$, a figure is required necessarily (see Fig 1 ).

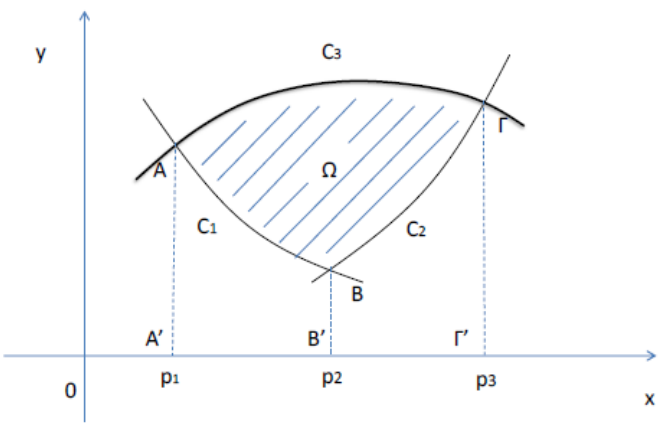

Fig 1

This work calculates the area that we described without requiring to draw and without finding any sign. Specifically, there will be no need in finding the sign for the functions $f_{3}-f_{1}, f_{3}-f_{2}$ and the drawing of $\mathrm{C}_{\mathrm{f}_{1}}, \mathrm{C}_{\mathrm{f}_{2}}, \mathrm{C}_{\mathrm{f}_{3}}$ for the calculation of $\mathrm{E}(\Omega)$.

III. Calculating the level region $(\Omega)$ using continuous functions The area of the region $(\Omega)$ is given by the formula:

$\mathrm{E}(\Omega)=\left|\int_{\mathrm{p}_{1}}^{\mathrm{p}_{2}} \mathrm{f}_{1} \mathrm{x}+\int_{\mathrm{p}_{2}}^{\mathrm{p}_{3}} \mathrm{f}_{2}(\mathrm{x}) \mathrm{dx}+\int_{\mathrm{p}_{3}}^{\mathrm{p}_{1}} \mathrm{f}_{3}(\mathrm{x}) \mathrm{dx}\right|(1)$

because if

- $\left(\Omega_{1}\right)$ is the region which is enclosed by $\mathrm{C}_{1}$, the straight lines $\mathrm{x}=\mathrm{p}_{1}, \mathrm{x}=\mathrm{p}_{2}$ and the axis $\mathrm{x}$ 'x

- $\left(\Omega_{2}\right)$ is the region area which is enclosed by $\mathrm{C}_{2}$, the straight lines $\mathrm{x}=\mathrm{p}_{2}, \mathrm{x}=\mathrm{p}_{3}$ and the axis $\mathrm{x}$ ' $\mathrm{x}$ and

- $\left(\Omega_{3}\right)$ is the region which is enclosed by $C_{3}$, the straight lines $\mathrm{x}=\mathrm{p}_{1}, \mathrm{x}=\mathrm{p}_{3}$ and the axis x'x

as it is evident from Figure (1) we have:

$$
\mathrm{E}(\Omega)=\left|\mathrm{E}\left(\Omega_{1}\right)+\mathrm{E}\left(\Omega_{2}\right)-\mathrm{E}\left(\Omega_{3}\right)\right|=\left|\int_{\mathrm{p}_{1}}^{\mathrm{p}_{2}} \mathrm{f}_{1}(\mathrm{x})+\int_{\mathrm{p}_{2}}^{\mathrm{p}_{3}} \mathrm{f}_{2}(\mathrm{x}) \mathrm{dx}-\int_{\mathrm{p}_{1}}^{\mathrm{p}_{3}} \mathrm{f}_{3}(\mathrm{x}) \mathrm{dx}\right|=
$$




$$
=\left|\int_{p_{1}}^{p_{2}} f_{2}(x) d x+\int_{p_{2}}^{p_{3}} f_{1}(x)+\int_{p_{3}}^{p_{1}} f_{3}(x) d x\right|
$$

It is not required for $\mathrm{f}_{1}(\mathrm{x})>0, \mathrm{f}_{2}(\mathrm{x})>0, \mathrm{f}_{3}(\mathrm{x})>0$ to apply, as there will always be a $\mathrm{c} \in \mathfrak{R}$ that : $\mathrm{f}_{1}(\mathrm{x})+\mathrm{c}>0, \mathrm{f}_{2}(\mathrm{x})+\mathrm{c}>0, \mathrm{f}_{3}(\mathrm{x})+\mathrm{c}>0$

and the position of $f_{1}(x), f_{2}(x), f_{3}(x)$ in the formula that the previous functions take.

\subsection{Application mechanism for the formula 2}

In order to remember the formula (2) we write in turn the $\mathrm{p}_{1}, \mathrm{p}_{2}, \mathrm{p}_{3}$ and under every root, we denote the equation that it derives from (see fig 2 ).

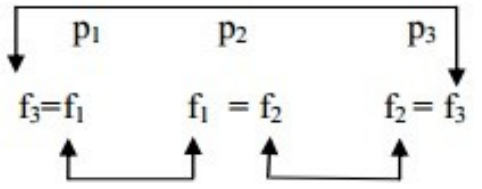

Fig. (2)

The area is equal with the absolute value of the sum of the three integrals with the

- First integral from $p_{1}$ to $p_{2}$ of the common function $f_{1}$ (see fig (2))

- Second integral from $\mathrm{p}_{2}$ to $\mathrm{p}_{3}$ of the common function $\mathrm{f}_{2}$ (see fig (2))

- Third integral from $p_{3}$ to $p_{1}$ of the common function $f_{3}$ (see fig (2))

Thus we don't need to draw $C_{1}, C_{2}, C_{3}$ and we don't need to find the sign of the differences $f_{2}-f_{1}, f_{2}-f_{3}$ which was required until now by the formula (1).

\subsection{Application}

Calculate the area of level region $(\Omega)$ which is enclosed by the graphs of the functions

$\mathrm{f}_{1}(\mathrm{x})=\ln \frac{1}{\mathrm{x}}=-\ln \mathrm{x}$

$f_{2}(x)=\ln x$

$f_{3}(x)=\ln 2$

Solution

$\mathrm{f}_{3}(\mathrm{x})=\mathrm{f}_{1}(\mathrm{x}) \Leftrightarrow \ln \frac{1}{2}=\ln \mathrm{x} \Leftrightarrow \mathrm{x}=\frac{1}{2}$

$\mathrm{f}_{1}(\mathrm{x})=\mathrm{f}_{2}(\mathrm{x}) \Leftrightarrow \ln \frac{1}{\mathrm{x}}=\ln \mathrm{x} \Leftrightarrow \mathrm{x}=1$

$\mathrm{f}_{2}(\mathrm{x})=\mathrm{f}_{3}(\mathrm{x}) \Leftrightarrow \ln \mathrm{x}=\ln 2 \Leftrightarrow \mathrm{x}=2$

Therefore, $\mathrm{p}_{1}=\frac{1}{2}, \mathrm{p}_{2}=1, \mathrm{p}_{3}=2$

We draw the design that I mentioned in the memory rule

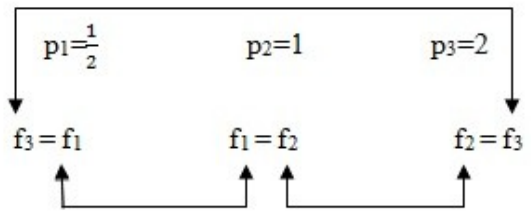

We have, without designing, the region $(\Omega)$ :

$\mathrm{E}(\Omega)=\int_{\frac{1}{2}}^{1} \ln \frac{1}{\mathrm{x}} \mathrm{dx}+\int_{1}^{2} \ln \mathrm{xdx}+\int_{2}^{\frac{1}{2}} \ln 2 \mathrm{dx}=\ldots=$

\section{Conclusion}

In this paper a different approach on area calculation of the level region was introduced. The method commonly used until now required the use of graphs and signs for the functions that were involved in the process whereas with the new way of calculating the area only continuous functions are used. It was proven to be easier and more accessible. By using this method, hopefully the calculation of such an area will be simpler and quicker.

[1]. Aligniac, G. 1995 Mathematics, Athens, Aithrio

References

[2]. Apostol, T 1962 differential and integral calculus, Athens, Atlantis

[3]. Brand, L. 1984 Mathematical Analysis, Athens E.M.E.

[4]. Boyce, W. \& Diprima, R. (1999) Elementary differential equations and boundary value problems, NTUA

[5]. Rudin, W. (2000) Principles of Mathematical Analysis, athens, Leader Books

[6]. Spiegel, M. (1982) higher Mathematics, ESPI

[7]. Thomas, G.B. \& Finney, R.L. (2001) Advanced Calculus, Volume A, Crete 